\title{
Establishment and validation of an individualized nomogram to predict distant metastasis in chondrosarcoma patients: a population-based study
}

\author{
Tien-Manh Hoang ${ }^{1 \#}$, Minh-Tien Nguyen ${ }^{1,2 \#}$, Weisin Chen ${ }^{1}$, Chenyang Zhuang ${ }^{1}$, Zixiang Wang ${ }^{1}$, \\ Hanquan Wang ${ }^{1}$, Juan $\mathrm{Li}^{1,3}$, Hong Lin ${ }^{1,3}$
}

${ }^{1}$ Department of Orthopedics, Zhongshan Hospital, Fudan University, Shanghai, China; ${ }^{2}$ Department of Spinal Surgery, Institute of Trauma and Orthopedics, 108 Military Central Hospital, Hanoi, Vietnam; ${ }^{3}$ Department of Orthopedics, Shanghai Geriatrics Center, Fudan University, Shanghai, China

Contributions: (I) Conception and design: J Li, H Lin; (II) Administrative support: H Lin; (III) Provision of study materials or patients: J Li; (IV) Collection and assembly of data: TM Hoang, MT Nguyen, W Chen; (V) Data analysis and interpretation: TM Hoang, MT Nguyen, C Zhuang; (VI) Manuscript writing: All authors; (VII) Final approval of manuscript: All authors.

"These authors contributed equally to this work.

Correspondence to: Juan Li; Hong Lin. Department of Orthopedics, Zhongshan Hospital, Fudan University, No. 180 Fenglin Road, Shanghai 200032, China; Department of Orthopedics, Shanghai Geriatrics Center, Fudan University, No. 2560 Chunshen Road, Shanghai 201104, China.

Email: li_juan1983@outlook.com; lin.hong1@zs-hospital.sh.cn.

Background: Distant metastasis is a significant factor influencing chondrosarcoma (CHS) patients' treatment and prognosis. We aimed to establish a consistent and effective nomogram to better predict distant metastases of CHS individuals.

Methods: The Surveillance, Epidemiology and End Results (SEER) database was used to obtain the demographics and clinicopathological characteristics of CHS patients from 2010 to 2018. Independent risk factors were identified via univariate and multivariate logistic regressive analysis. A nomogram that predicts metastasis risk was established based on the training cohort, and its accuracy was validated through the validation cohort. The performance of this predictive model was assessed by the receiver operating characteristic (ROC) curve and Harrell's concordance index (C-index). Finally, decision curve analysis (DCA) was conducted to test its clinical reliability.

Results: Data of 1,066 patients were extracted, of these, 66 cases (6.19\%) were with distant metastasis at initial diagnosis. The following features were shown to be linked to an increased risk of metastasis: highgrade tumor, T3 stage, and large tumor size; whereas unmarried and use of surgery were independent protective factors. Marital status, tumor grade, $\mathrm{T}$ stage, use of cancer-directed surgery and tumor size were incorporated to develop the novel nomogram. The ROC curves showed the effectiveness of the nomogram with the high area under the curves, the C-indices were 0.931 and 0.951 in the internal and external validation, respectively. The calibration plots indicated a good consistency and agreement of the nomogram, while the DCA illustrated that the nomogram had favorable potential clinical applicability due to great positive net benefit with wide ranges of the threshold probabilities.

Conclusions: This work developed a novel nomogram for predicting distant metastasis in CHS patients, which might assist clinicians to determine the optimal treatment plan by precisely predicting individualized metastatic risk.

Keywords: Chondrosarcoma (CHS); metastasis; risk factors; nomogram; Surveillance, Epidemiology and End Results (SEER)

Submitted Oct 12, 2021. Accepted for publication Jan 19, 2022.

doi: $10.21037 /$ tcr-21-2212

View this article at: https://dx.doi.org/10.21037/tcr-21-2212 


\section{Introduction}

In 2021, an expected 3,610 persons were diagnosed with primary bone malignancies, with an anticipated 2060 fatalities due to these tumors in the United States (US) (1). Chondrosarcoma (CHS) is the second most prevalent bone malignancy after osteosarcoma (35\%), accounting for 30\% of primary bone sarcomas (2). Histologically, CHSs are characterized by the formation of a non-osteoid cartilage matrix by tumor cells. Both chemo- and radiotherapy have little efficacy due to the plenteous extracellular matrix, limited vascularity, less cellular dividing, and sluggish growth of CHS $(3,4)$, hence the most universally accepted treatment is a wide resection with appropriate surgical margins (5). It is widely assumed that metastasis is a significant factor influencing CHS patients' treatment and prognosis. The treatment of individuals with and without distant metastasis differs significantly. Since metastasis is certainly multifactorial, impacted by a variety of factors, finding methods to combine all available data to arrive at a more exact estimate appears to be critical.

By combining multiple potential risks, nomograms have been utilized as user-reliable and helpful statistical tools to predict the probability of a given outcome in various malignancies, such as thyroid carcinoma (6), bladder cancer (7), renal cell carcinoma (8), pancreatic ductal adenocarcinoma (9), and gastrointestinal stromal tumor (10). However, to the best of our knowledge, no nomograms for predicting distant metastases in CHS patients have been constructed. Therefore, the goal of this study was to establish a consistent and effective nomogram based on a large population to better predict distant metastases of CHS individuals.

We present the following article in accordance with the TRIPOD reporting checklist (available at https://tcr. amegroups.com/article/view/10.21037/tcr-21-2212/rc).

\section{Methods}

\section{Study population}

CHS patient data were obtained from the Surveillance, Epidemiology, and End Results (SEER) Program of the National Cancer Institute with SEER $*$ Stat software version 8.3.9.1 (National Cancer Institute, Bethesda, USA). The SEER database is the largest open-access dataset covering approximately $30 \%$ of the US population (11). It collects demographic, clinicopathological, and survival information of cancer patients from 18 population-based cancer registries. The complete information on distant metastases was only available from 2010 and onward, hence this study was restricted to the period between 2010 and 2018. The study was conducted in accordance with the Declaration of Helsinki (as revised in 2013). Since the data from the SEER registry were de-identified and publicly available, no institutional review board approval was necessary and no informed consent was signed for this study.

Our research cohort consisted of subjects from 18 cancer registries of the database with the following inclusion criteria: (I) histology code served as an indication of CHS, which was 9220, 9221, 9230, 9231, 9240, 9242, and 9243, according to the International Classification of Diseases for Oncology, 3rd Edition (ICD-O-3) morphology codes; (II) CHS as the only primary cancer; (III) patients diagnosed between 2010 and 2018; (IV) diagnosis confirmed by pathology; (V) patients with clear information of the distant metastases. Exclusion criteria were as follows: patients with missing or incomplete information about age, race, sex, marital status, primary site, histological grade, $\mathrm{T}$ stage, $\mathrm{N}$ stage, surgery, and tumor size. Distant metastasis was the ultimate outcome evaluated in the present study.

\section{Demographics and clinicopathological characteristics}

Demographic characteristics of patients included age at diagnosis, race, sex, and marital status. Patients' race was stratified into three groups: White, Black, and others, which included American Indian/Alaska Native and Asian/ Pacific Islander. Marital status was classified as married or unmarried. It should be noted that the unmarried status included single (never married), separated, divorced, widowed, or domestic partner.

Clinicopathological characteristics included primary tumor site, tumor grade, $\mathrm{T}$ stage, $\mathrm{N}$ stage, surgery, and tumor size. Since the SEER database did not show the details of the anatomic site, for example, the cervical vertebrae, thoracic vertebrae, or lumbar vertebrae were recorded as vertebral column without distinction, primary sites were classified into three groups: extremity (C40.03, C40.8, C40.9), pelvis/spine (C41.2-4), and skull (C41.0, $\mathrm{C} 41.1)$. We regrouped the tumor grade into low grade (included well-differentiated lesions and moderately differentiated lesions) and high grade (included poorly differentiated lesions and undifferentiated and anaplastic lesions) (12). Whereas, the classification of $\mathrm{T}$ stage and $\mathrm{N}$ stage was based on the American Joint Committee on Cancer (AJCC) 7th Edition and the SEER Combined stage. 
Surgery status served as a surgical procedure that destroyed and/or removed tissue of the primary CHS performed as part of the first course or initial workup of treatment. Regarding tumor size variable, it notes the biggest dimension of the primary tumor in centimeters.

\section{Statistical analysis}

Kaplan-Meier survival analysis and log-rank test was performed between patients with and without distant metastasis. We randomly divided all patients $(\mathrm{N}=1,066)$ into two cohorts, training cohort and validation cohort, with a ratio of 70:30 to establish and validate the nomogram, respectively $(13,14)$. The Pearson's chi-squared test was used to compare categorical variables, and the Student's $t$-test was used to compare quantitative variables between the training cohort and the validation cohort.

Univariate logistic regression analysis was applied to discover potential risk factors of distant metastases. These factors with $\mathrm{P}$ values less than 0.05 were further integrated into the multivariate logistic regression analysis, then the independent risk factors in CHS patients were identified. We used the odds ratio (OR) and corresponding 95\% confidence interval (CI) to show the relationship between these independent risk factors and distant metastases.

Based on the consequences confirmed from the logistic analyses, we constructed a nomogram via the training cohort. Meanwhile, its accuracy was validated through the validation cohort. Internal validation (training cohort) and external validation (validation cohort) were applied with 1,000 bootstrap resamples to prevent overfitting as well as to avoid achieving a biased estimation. We assessed the performance of this predictive model by receiver operating characteristic (ROC) curve (15) and Harrell's concordance index (C-index) (16). C-index takes a value between 0.5 and 1.0 , the minimum value 0.5 indicates random chance and the maximum value 1.0 indicates a perfectly corrected discrimination. A value of C-index greater than 0.7 is considered a relatively accurate prediction. Calibration plots were generated to compare nomogram predictions with observed outcomes internally and externally $(16,17)$. Besides, decision curve analysis (DCA), which was a method for assessing alternative prognostic or diagnostic tools that had outstanding advantages than others (18), was conducted to test the clinical reliability of the novel model. Figure 1 shows the process of the patient selection and analyses in the present study.

All statistical tests in this study were performed using the
$\mathrm{R}$ programming language version 4.0.5 (The R Foundation for Statistical Computing, Vienna, Austria, http://www. r-project.org/). Two-tailed $\mathrm{P}$ values $<0.05$ were considered to be statistically significant in all statistical analyses.

\section{Results}

\section{Baseline characteristics}

According to the inclusion criteria and exclusion criteria outlined above, 1,066 patients were eligible to identify the risk factors of distant metastases in patients with CHS. Of these, 66 cases $(6.19 \%)$ were with distant metastasis at initial diagnosis, and 1,000 cases $(93.81 \%)$ were not.

Patients' demographics and clinicopathological characteristics are listed in Table 1. The patients have a mean age of $51.41 \pm 17.21$ years. The total cohort was mostly White patients (931; 87.34\%), 73 (6.85\%) patients are Black, and 62 (5.82\%) are American Indian/Alaska Native or Asian/Pacific Islander. The gender distribution indicated that there was a tiny male predominance (54.97\%). Among these CHS subjects, 632 (59.29\%) were married, and 434 (40.71\%) were unmarried. Regarding primary tumor site, extremities and pelvis/spine were the majority and had approximately the same ratios $(49.44 \%$ and $42.03 \%$, respectively), and skull only accounted for a small percentage (8.54\%). The majority was low grade in tumor grade distribution (76.36\%). About the T stage, T1, T2 and T3 accounted for $60.79 \%, 37.05 \%$ and $2.16 \%$, respectively. The $\mathrm{N}$ stage was divided into N0 (98.59\%) and N1 (1.41\%). Most patients did not have cancer-directed surgery $(93.62 \%)$. The mean tumor size of the study population was $7.53 \pm 7.51 \mathrm{~cm}$.

The total cohort was randomly assigned into the training cohort (746 cases) and the validation cohort (320 cases), with a ratio of approximately 7:3. As shown in Table 1, the training and validation cohort had no significant differences regarding patients' age, race, sex, marital status, primary site, tumor grade, $\mathrm{T}$ stage, $\mathrm{N}$ stage, use of surgery, tumor size, and metastatic status $(\mathrm{P}>0.05)$.

\section{Kaplan-Meier survival analysis and metastasis-related factors}

The Kaplan-Meier survival curve was shown in Figure 2. The result of the log-rank test showed that CHS patients with distant metastases are significantly poor than that of CHS patients without distant metastasis regarding the overall survival (OS). 


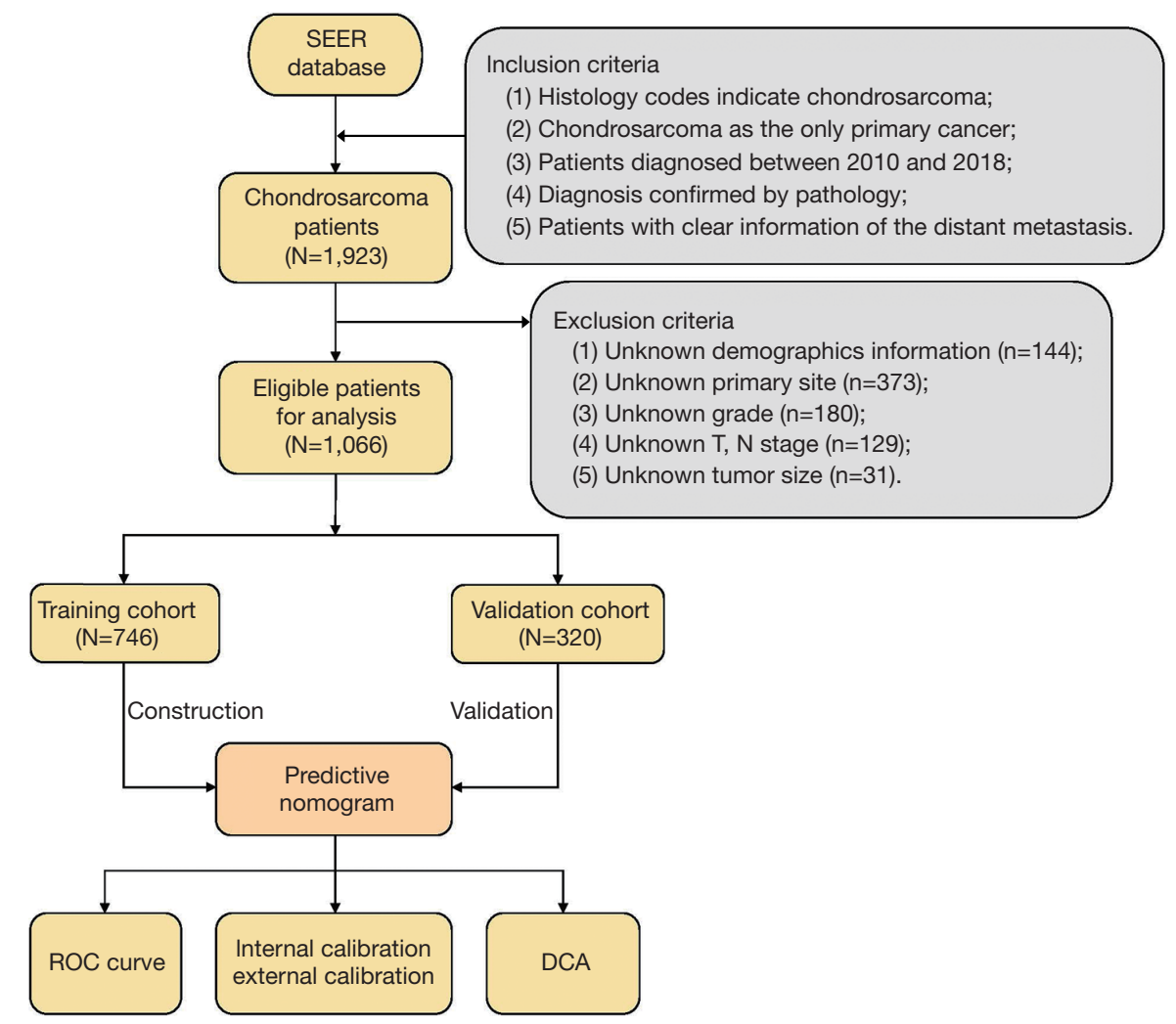

Figure 1 Flow diagram of the process of the patient selection and analyses in the present study. 1,923 patients were initially gathered from the SEER database, and 857 ineligible patients were excluded based on the exclusion criteria, then 1,066 eligible patients were randomly divided into the training [746] and validation [320] set. SEER, Surveillance, Epidemiology and End Results; ROC, receiver operating characteristic; DCA, decision curve analysis.

To investigate the independent risk factors, logistic regressive analysis was performed. On the basis of univariate analysis, significant differences of several variables were shown between patients with and without distant metastasis, including age at diagnosis, marital status, grade, $\mathrm{T}$ stage, $\mathrm{N}$ stage, cancer-directed surgery, and tumor size (all $\mathrm{P}$ values $<0.05$ ). Multivariate logistic regression analysis was further performed to control for confounding variables. The multivariate regression demonstrated that high-grade tumor $(\mathrm{OR}=10.163,95 \% \mathrm{CI}: 4.098-25.202, \mathrm{P}<0.001)$, T3 stage ( $\mathrm{OR}=11.864,95 \%$ CI: 2.258-62.325, $\mathrm{P}=0.003)$, and tumor size $(\mathrm{OR}=1.055,95 \% \mathrm{CI}$ : $1.010-1.101, \mathrm{P}=0.016)$ were independent risk factors, whereas unmarried $(\mathrm{OR}=0.268$, 95\% CI: $0.098-0.733, \mathrm{P}=0.010)$ and use of surgery (OR $=0.037,95 \%$ CI: $0.013-0.100, \mathrm{P}<0.001)$, were independent protect factors. The results of the logistic regressive analysis are illustrated in Table 2.

\section{Construction and validation of a novel nomogram}

After selection, marital status, tumor grade, $\mathrm{T}$ stage, use of cancer-directed surgery and tumor size were incorporated to develop the novel nomogram which predicted the risk of distant metastases in CHS patients (Figure 3). The point for each predictor is determined by the patient's specific status for that predictor. Detailed points of nomogram predictors were illustrated in Table 3. The nomogram is used in the following way: we first calculate the total points based on all of the single points, then we create a line perpendicular to the Total points bar and the Risk bar to define the probability, which are between $1 \%$ and $90 \%$.

In both the training and validation sets, the ROC curves showed the effectiveness of the nomogram with the high area under the curves (Figure 4). The C-indices were 0.931 in the internal validation, and 0.951 in the external validation. Both internal calibration and external calibration 
Table 1 Baseline characteristics of patients with chondrosarcoma

\begin{tabular}{|c|c|c|c|c|}
\hline Characteristics & Total $(n=1,066)$ & Training cohort $(n=746)$ & Validation cohort $(n=320)$ & $P$ value $^{\dagger}$ \\
\hline Race, n (\%) & & & & 0.444 \\
\hline White & $931(87.34)$ & $653(87.53)$ & $278(86.88)$ & \\
\hline Black & $73(6.85)$ & $47(6.3)$ & $26(8.12)$ & \\
\hline Sex, n (\%) & & & & 0.353 \\
\hline Male & $586(54.97)$ & $417(55.90)$ & $169(52.81)$ & \\
\hline Female & $480(45.03)$ & $329(44.10)$ & $151(47.19)$ & \\
\hline Marital status, n (\%) & & & & 0.294 \\
\hline Primary site, n (\%) & & & & 0.278 \\
\hline Extremity & $527(49.44)$ & $373(50.00)$ & $154(48.12)$ & \\
\hline Pelvis/spine & $448(42.03)$ & $316(42.36)$ & $132(41.25)$ & \\
\hline Skull & $91(8.54)$ & $57(7.64)$ & $34(10.62)$ & \\
\hline Grade, n (\%) & & & & 0.832 \\
\hline Low & $814(76.36)$ & $571(76.54)$ & $243(75.94)$ & \\
\hline High & $252(23.64)$ & $175(23.46)$ & $77(24.06)$ & \\
\hline T stage, n (\%) & & & & 0.519 \\
\hline No & $1,051(98.59)$ & $736(98.66)$ & $315(98.44)$ & \\
\hline N1 & $15(1.41)$ & $10(1.34)$ & $5(1.56)$ & \\
\hline Surgery, n (\%) & & & & 0.910 \\
\hline No & $998(93.62)$ & $698(93.57)$ & $300(93.75)$ & \\
\hline Yes & $68(6.38)$ & $48(6.43)$ & $20(6.25)$ & \\
\hline Size $(\mathrm{cm})$ & $7.53 \pm 7.51$ & $7.63 \pm 7.87$ & $7.3 \pm 6.62$ & 0.476 \\
\hline Metastasis, n (\%) & & & & 0.544 \\
\hline No & $1,000(93.81)$ & $702(94.10)$ & $298(93.12)$ & \\
\hline Yes & $66(6.19)$ & $44(5.90)$ & $22(6.88)$ & \\
\hline
\end{tabular}

\footnotetext{
${ }^{\dagger}$, between-group differences were compared by Pearson's chi-squared test or Student's $t$-test, respectively.
} 

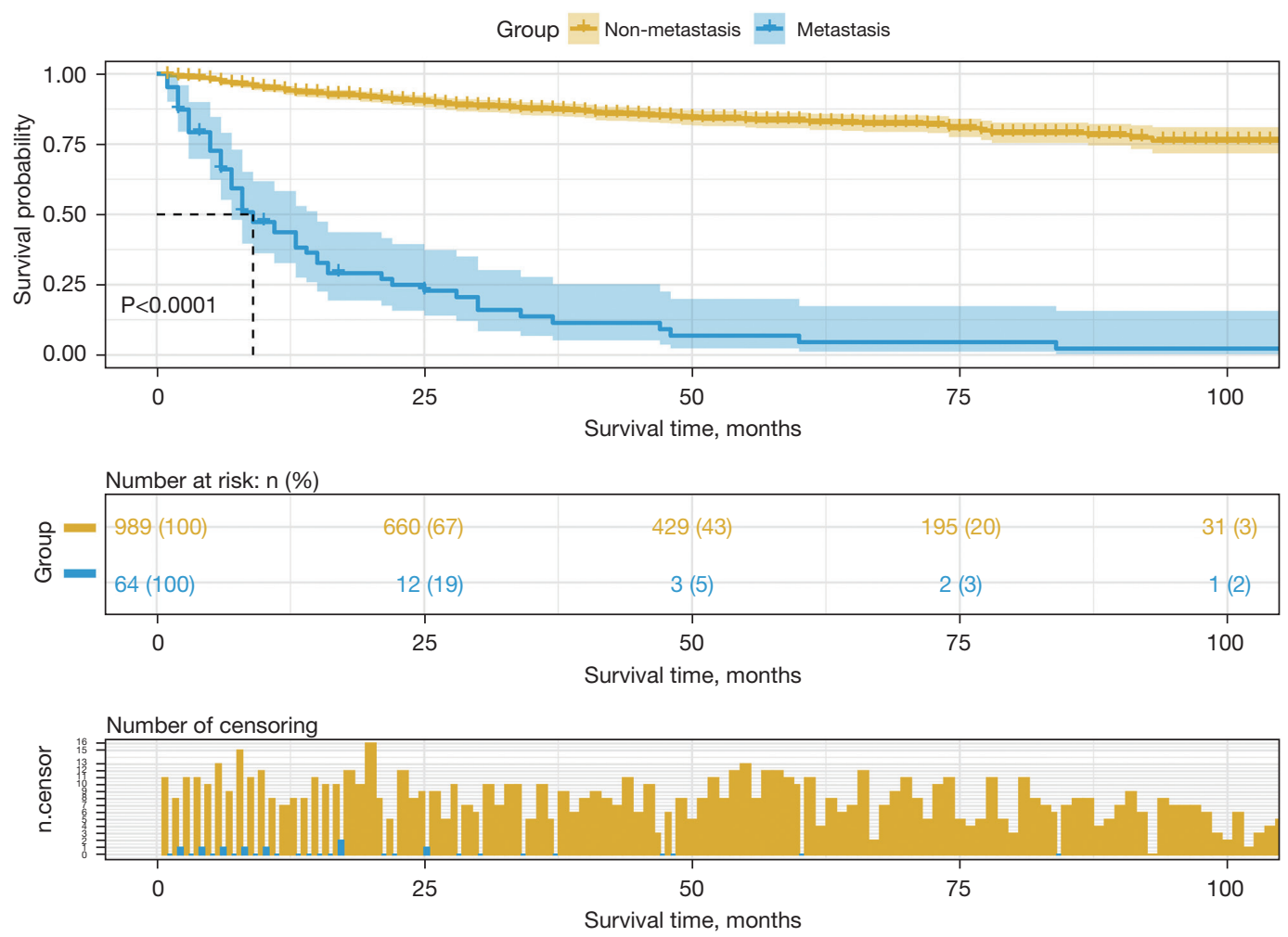

Figure 2 Kaplan-Meier analysis for overall survival in metastatic and non-metastatic chondrosarcoma.

plots indicated a good consistency and agreement between nomogram-predicted probability and actual probability of distant metastases (Figure 5). Besides, the DCA (Figure 6) illustrated that the nomogram had favorable potential clinical applicability in predicting the risk of distant metastases due to great positive net benefit with wide ranges of the threshold probabilities.

\section{Discussion}

In the present study, there was $6.19 \%$ of CHS patients had distant metastasis at initial diagnosis, whereas, according to a large population-based comparative study conducted by Giuffrida et al. (19), the percentage was approximately $8 \%$. This discrepancy might be attributable to our study's inclusion of patient data after 2010, which was not accessible at the study time of Giuffrida et al.

Distant metastasis is thought to be a common sign of advanced stages in most cancers, including CHS, and according to prior reports, CHS patients with metastasis had a poorer prognosis than those without metastasis $(5,20-23)$. van Maldegem et al. (22) stated that patients with the unresectable, locally advanced disease without distant metastasis had significantly better survival than patients with metastatic disease $(\mathrm{P}=0.0014)$. In another research, Andreou et al. (20) found that patients with metastases had a 5 -year survival rate of only $23 \%$, whereas it was $89 \%$ in those without metastatic disease. Song et al. (23) indicated that distant metastasis was the independent meaningful risk factor for both OS and cancer-specific survival (CSS) in CHS patients [hazard ratio (HR) $=4.483$, 95\% CI: $3.015-$ 6.666; HR =5.367, 95\% CI: 3.469-8.302, respectively].

However, prior studies have only looked on risk variables that impact the prognosis of CHS patients $(24,25)$. There is yet to be a study that focuses on finding the risk of CHS metastasis, which is one of the most crucial elements in the cancer prognosis. Besides, as a quantitative tool for evaluating hazard and benefit, nomograms have been extensively used in the medical field for clinical decisionmaking purposes (26), several nomograms for predicting the OS and CSS of CHS have been constructed and validated in earlier research $(23,27,28)$. The nomogram for predicting CHS distant metastases, on the other hand, has yet to be reported. Therefore, we developed a unique 
Table 2 Univariate and multivariate logistic regression analysis in the training cohort

\begin{tabular}{|c|c|c|c|}
\hline \multirow{2}{*}{ Characteristics } & \multirow{2}{*}{$\begin{array}{c}\text { Univariate } \\
\text { analysis }\end{array}$} & \multicolumn{2}{|c|}{ Multivariate analysis } \\
\hline & & OR (95\% Cl) & $P$ value \\
\hline Age & 0.001 & $1.018(0.992-1.045)$ & 0.178 \\
\hline Race & 0.723 & $\mathrm{NI}$ & \\
\hline White & & & \\
\hline Black & & & \\
\hline Other & & & \\
\hline Sex & 0.453 & $\mathrm{NI}$ & \\
\hline Female & & & \\
\hline Male & & & \\
\hline Marital status & 0.010 & & \\
\hline Married & & $\mathrm{R}$ & \\
\hline Unmarried & & $0.268(0.098-0.733)$ & 0.010 \\
\hline Primary site & 0.408 & $\mathrm{NI}$ & \\
\hline Extremity & & & \\
\hline Pelvis/spine & & & \\
\hline Skull & & & \\
\hline Grade & $<0.001$ & & \\
\hline Low & & $\mathrm{R}$ & \\
\hline High & & 10.163 (4.098-25.202) & $<0.001$ \\
\hline T stage & $<0.001$ & & \\
\hline $\mathrm{T} 1$ & & $\mathrm{R}$ & \\
\hline $\mathrm{T} 2$ & & $1.831(0.650-5.153)$ & 0.252 \\
\hline T3 & & $11.864(2.258-62.325)$ & 0.003 \\
\hline $\mathrm{N}$ stage & 0.005 & & \\
\hline NO & & $\mathrm{R}$ & \\
\hline N1 & & 3.746 (0.694-20.223) & 0.125 \\
\hline Surgery & $<0.001$ & & \\
\hline No & & $\mathrm{R}$ & \\
\hline Yes & & $0.037(0.013-0.100)$ & $<0.001$ \\
\hline Size & $<0.001$ & 1.055 (1.010-1.101) & 0.016 \\
\hline
\end{tabular}

OR, odds ratio; $\mathrm{Cl}$, confidence interval; $\mathrm{NI}$, not included; $\mathrm{R}$, reference. and comprehensive nomogram for predicting metastasis in CHS patients based on the SEER database (Figure 2), which may be applied in the clinical setting with patient-specific characteristics likely to be provided to the oncologists and orthopedic doctors. In the present study, we have built a logistic regression model to adjust for confounding variables and choose independent risk factors. Grade, tumor size, $T$ stage, cancer-directed surgery, and marital status were eventually identified as five contributors to distant metastases.

First, we found that histologic grade was an independent risk factor for metastasis, with individuals with high-grade tumors having a greater likelihood of developing distant metastases, this finding was consistent with those of prior research. According to Giuffrida et al. (19), the rate of survival at 30 years in the low-grade group was significantly higher than that in the high-grade group (76\% and $50 \%$, respectively, $\mathrm{P}<0.05$ ), in addition, patients in the highgrade group had an elevated death rate in the first decade, after that the survival began to stabilize. According to Thorkildsen et al. (29), high-grade tumors (II or III) were linked to higher risks of metastasis (grade II $v s$. grade I: HR $=3.90$; grade III $v s$. grade I: $\mathrm{HR}=16.46, \mathrm{P}<0.05)$. In highgrade malignancies, the structures of tumor cells and tissues are vastly abnormal-looking, as a result, it is generally more likely to develop and extend. Therefore, a higher histologic grade signifies a higher chance of metastasis.

Numerous earlier studies have demonstrated that large tumor is an independent negative factor in survival $(12,25,28,30)$, at the same time, it has a significant impact on metastatic development in CHS. However, the cutoff value was quite diverse between research. Puri et al. indicated that CHS patients with tumors $>8 \mathrm{~cm}$ exhibited a greater risk of local recurrence as well as had worse event-

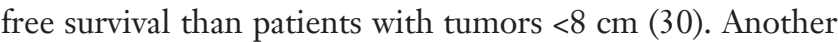
research chose 8.6 and 15.0 as two cut-off values; compared with patients with tumors smaller than $8.6 \mathrm{~cm}$, patients with tumors larger than $15.0 \mathrm{~cm}$ had a significantly higher risk of developing lung metastases $(\mathrm{P}=0.038)$, with an $\mathrm{OR}$ of 2.259 (1.044-4.884) (25). Considering that converting continuous variables to categorical variables might lose a lot of crucial information from the original data, we used tumor size as a continuous variable to investigate its effect on the 


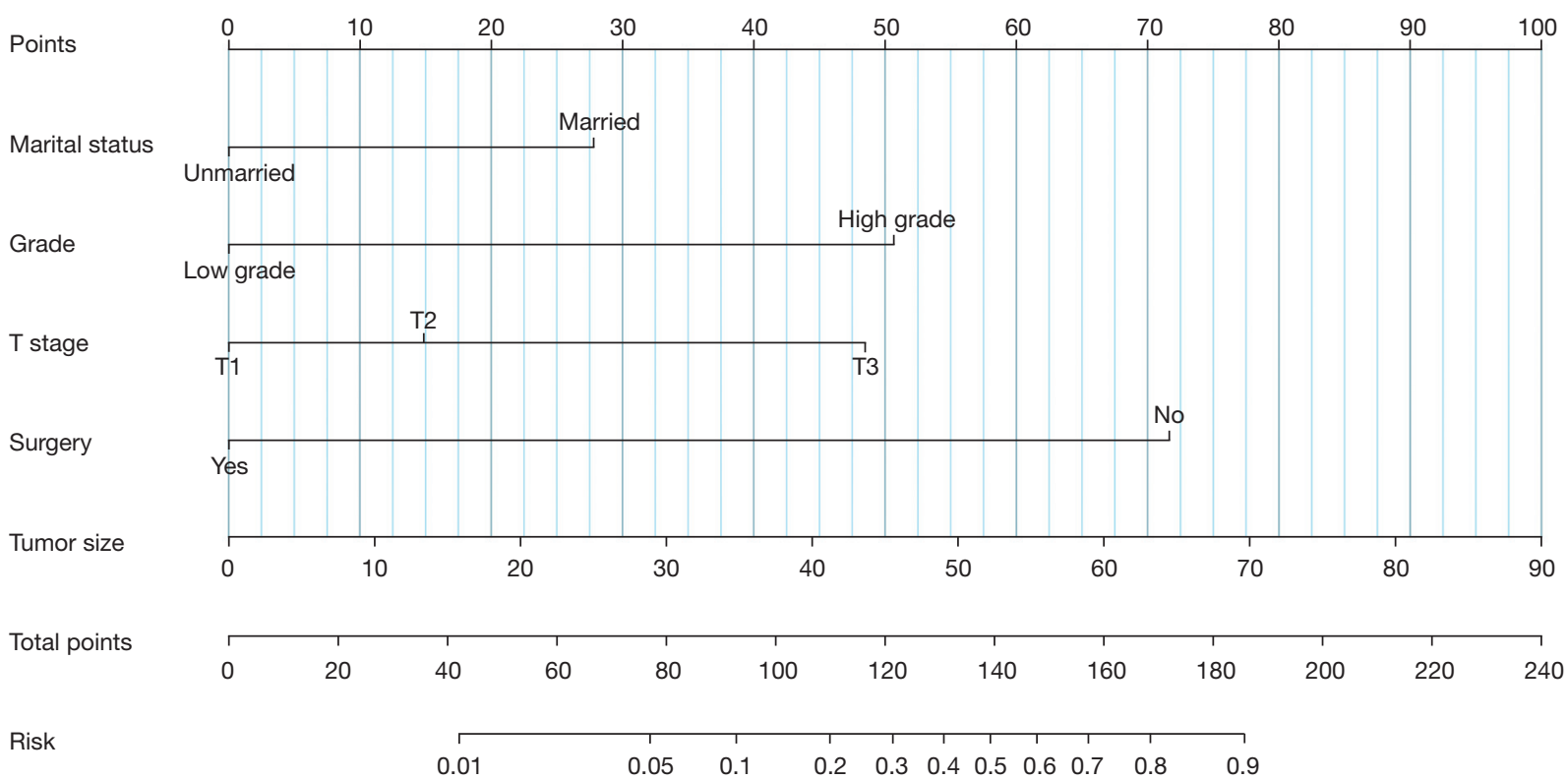

Figure 3 Nomogram predicting the risk of distant metastasis of CHS patients. Each factor's points were determined by creating a vertical line between it and the Points scale. We compute the metastatic risk by adding the points from each factor and creating a vertical line between the Total points scale and the Risk scale. CHS, chondrosarcoma.

Table 3 Detailed scores of each factor in the nomogram

\begin{tabular}{lcc}
\hline Characteristics & Classification & Points \\
\hline Marital status & Unmarried & 0 \\
Grade & Married & 28 \\
& Low & 0 \\
T stage & High & 51 \\
& T1 & 0 \\
Surgery & T2 & 15 \\
& T3 & 48 \\
Size $(\mathrm{cm})$ & Yes & 0 \\
\hline
\end{tabular}

probability of developing de novo metastases. The present study demonstrated that the OR increased $5.5 \%$ for every 1 -centimeter increase in tumor size. A large tumor may exert pressure on surrounding tissues and organs, raising the risk of local invasion and complicating primary tumor resection and appropriate margin achieving, thus increasing the possibility of distant metastasis.

Besides, logistic regression analysis also showed that $T$ stage was independently associated with a higher risk of distant metastases. In addition to informing how large the tumor is, $T$ stage is superior to tumor size in that it can also provide clinicians about where the tumor is and whether it's spread to surrounding tissues. The higher the tumor stage, the larger the tumor and/or the more it's spread to nearby tissues, thus the association between tumor stage and metastasis appears to be reasonable and logical.

In addition, cancer-directed surgery was also constituted to be a risk factor for distant metastases. Primary tumor surgery is the most common therapy for CHS, a wide resection with appropriate surgical margins slows the progression of malignancies, including metastasis. Despite the fact that the surgery effect is influenced by a variety of factors and the lack of surgery-related information in the SEER database such as surgical margin, blood loss, operative time, and transfusion rate, our findings suggested that CHS patients could benefit from resection of the primary tumor and that those who underwent surgery had a significantly lower metastasis rate.

It was notable that marital status was also an independent meaningful factor in the present study. Marital status has been reported as an independent prognostic factor on the prognosis of many malignancies, and marriage appeared to have a protective effect in these studies, with married 

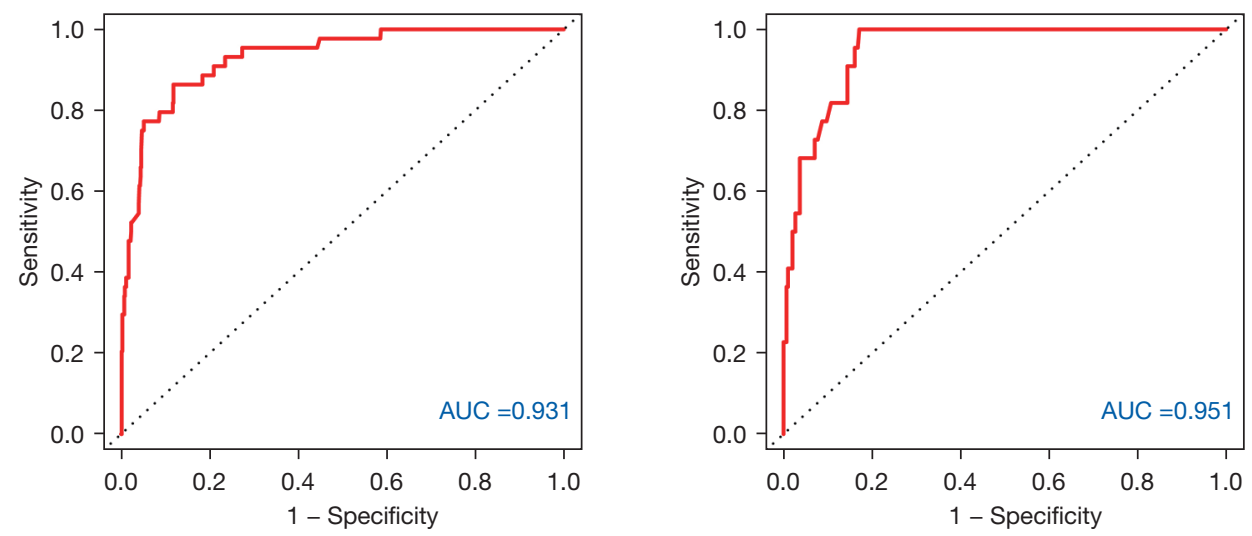

Figure 4 ROC curves of the Nomogram for predicting metastases in the training (A) and validation (B) sets. The AUCs were 0.931 in the training set, and 0.951 in the validation set. ROC, receiver operating characteristic; AUC, area under the curve.
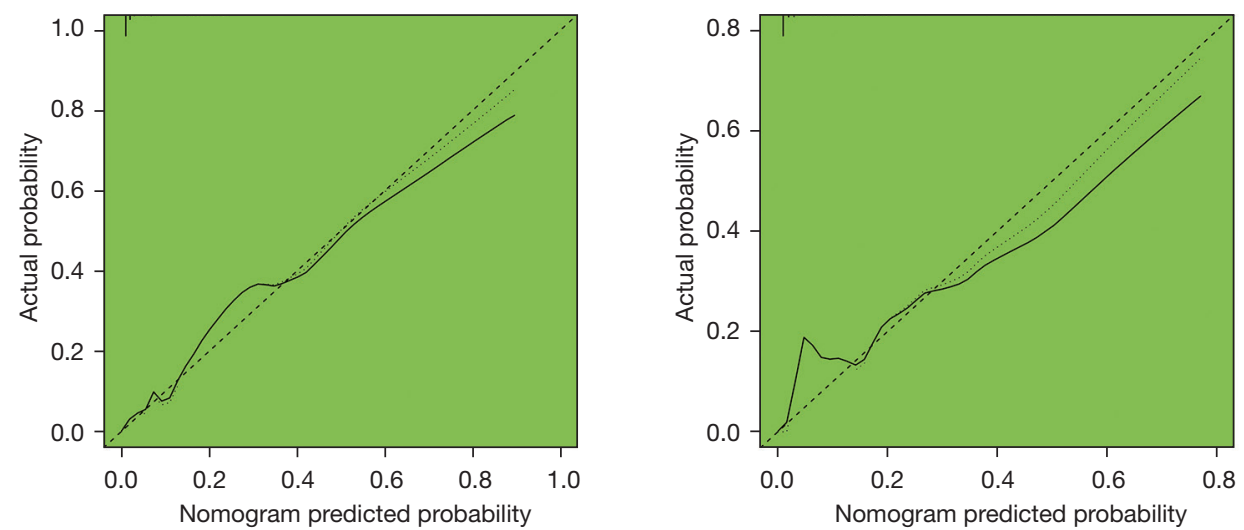

Figure 5 Plots depicting internal (A) and external (B) calibration diagrams. The $\mathrm{x}$-axis depicts the nomogram-predicted probability and the $\mathrm{y}$-axis depicts the actual probability in the calibration curve.

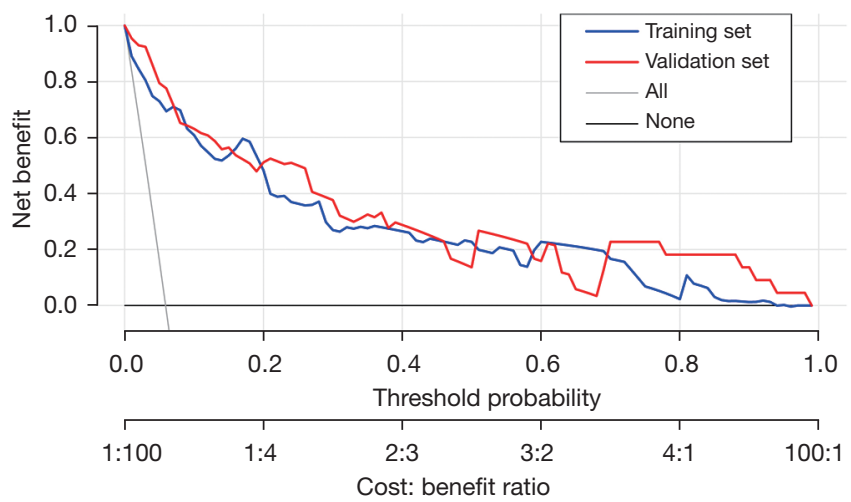

Figure 6 DCA for the Nomogram for predicting metastases in the training and validation sets. DCA, decision curve analysis. 
patients having better 5-year OS and CSS outcomes than unmarried patients, including divorced/separated, widowed, and single patients (31-33). Compared with unmarried individuals, married individuals generally have more advantageous financial situations and emotional support from their husbands or wives, thus they will adhere better to treatment and have better outcomes. In addition, Gao et al. (34) found that widowed CHS patients had the worst CSS when compared to married, single, and divorced individuals. This poor prognosis might be explained by delayed diagnosis, a large portion of patients with low socioeconomic level, or a low proportion of patients who had surgery (35).

This study had several following limitations. First, selection bias was unavoidable because of the retrospective character of the study, and it is necessary to conduct prospective studies to confirm our findings. Second, only metastatic data from 2010 is available in the database for the four sites including brain, lung, liver, and bone. Several additional potentially important variables, such as patient clinical symptoms, laboratory data, tumor biomarkers, and surgery-related information such as surgical margin, blood loss, operative time, and transfusion rate could not be obtained from the database. Finally, a large-scale external cohort is required for further validating the nomogram.

\section{Conclusions}

To conclude, a novel nomogram for predicting distant metastasis in patients with CHS was established using a population-based cohort drawn from the SEER database. Based on the results of internal and external validation and the DCA curve, our nomogram could assist clinicians to determine the optimal treatment plan by precisely predicting individualized metastatic risk.

\section{Acknowledgments}

The authors would like to thank all members of the SEER Program for their contributions to the SEER database.

Funding: This work was supported by the Non-profit Central Research Institute Fund of Chinese Academy of Medical Sciences (No. 2020-JKCS-013), ThreeYear Initiative Plan for Strengthening Public Health System Construction in Shanghai (No. GWV-10.2XD11), and Natural Science Foundation of Shanghai (No. 21ZR1455800).

\section{Footnote}

Reporting Checklist: The authors have completed the TRIPOD reporting checklist. Available at https://tcr. amegroups.com/article/view/10.21037/tcr-21-2212/rc

Peer Review File: Available at https://tcr.amegroups.com/ article/view/10.21037/tcr-21-2212/prf

Conflicts of Interest: All authors have completed the ICMJE uniform disclosure form (available at https://tcr.amegroups. com/article/view/10.21037/tcr-21-2212/coif). All authors report grants from the Non-profit Central Research Institute Fund of Chinese Academy of Medical Sciences (No. 2020-JKCS-013), Three-Year Initiative Plan for Strengthening Public Health System Construction in Shanghai (No. GWV-10.2-XD11), and Natural Science Foundation of Shanghai (No. 21ZR1455800). The authors have no other conflicts of interest to declare.

Ethical Statement: The authors are accountable for all aspects of the work in ensuring that questions related to the accuracy or integrity of any part of the work are appropriately investigated and resolved. The study was conducted in accordance with the Declaration of Helsinki (as revised in 2013). Since the data from the SEER registry were de-identified and publicly available, no institutional review board approval was necessary and no informed consent was signed for this study.

Open Access Statement: This is an Open Access article distributed in accordance with the Creative Commons Attribution-NonCommercial-NoDerivs 4.0 International License (CC BY-NC-ND 4.0), which permits the noncommercial replication and distribution of the article with the strict proviso that no changes or edits are made and the original work is properly cited (including links to both the formal publication through the relevant DOI and the license). See: https://creativecommons.org/licenses/by-nc-nd/4.0/.

\section{References}

1. Siegel RL, Miller KD, Fuchs HE, et al. Cancer Statistics, 2021. CA Cancer J Clin 2021;71:7-33.

2. Biermann JS, Chow W, Reed DR, et al. NCCN Guidelines Insights: Bone Cancer, Version 2.2017. J Natl Compr Canc Netw 2017;15:155-67.

3. Bovée JV, Cleton-Jansen AM, Taminiau AH, et al. 
Emerging pathways in the development of chondrosarcoma of bone and implications for targeted treatment. Lancet Oncol 2005;6:599-607.

4. Delaney TF, Kepka L, Goldberg SI, et al. Radiation therapy for control of soft-tissue sarcomas resected with positive margins. Int J Radiat Oncol Biol Phys 2007;67:1460-9.

5. Nota SP, Braun Y, Schwab JH, et al. The Identification of Prognostic Factors and Survival Statistics of Conventional Central Chondrosarcoma. Sarcoma 2015;2015:623746.

6. Tong Y, Hu C, Huang Z, et al. Novel nomogram to predict risk of bone metastasis in newly diagnosed thyroid carcinoma: a population-based study. BMC Cancer 2020;20:1055.

7. Cai YL, Lin YX, Jiang LS, et al. A Novel Nomogram Predicting Distant Metastasis in T1 and T2 Gallbladder Cancer: A SEER-based Study. Int J Med Sci 2020;17:1704-12.

8. Tong Y, Huang Z, Hu C, et al. Construction and Validation of a Convenient Clinical Nomogram to Predict the Risk of Brain Metastasis in Renal Cell Carcinoma Patients. Biomed Res Int 2020;2020:9501760.

9. He C, Zhong L, Zhang Y, et al. Development and validation of a nomogram to predict liver metastasis in patients with pancreatic ductal adenocarcinoma: a large cohort study. Cancer Manag Res 2019;11:3981-91.

10. Zhou G, Xiao K, Gong G, et al. A novel nomogram for predicting liver metastasis in patients with gastrointestinal stromal tumor: a SEER-based study. BMC Surg 2020;20:298.

11. Surveillance, Epidemiology, and End Results Program [Internet]. Rockville (MD): National Cancer Institute; [cited 2021 Apr 02]. Available online: https://seer.cancer.gov/

12. Arshi A, Sharim J, Park DY, et al. Chondrosarcoma of the Osseous Spine: An Analysis of Epidemiology, Patient Outcomes, and Prognostic Factors Using the SEER Registry From 1973 to 2012. Spine (Phila Pa 1976) 2017;42:644-52.

13. Iasonos A, Schrag D, Raj GV, et al. How to build and interpret a nomogram for cancer prognosis. J Clin Oncol 2008;26:1364-70.

14. Li Q, Chen Q, Chen J, et al. Prognostic nomogram for predicting long-term survival in bronchopulmonary carcinoid tumor patients receiving resection. Ann Transl Med 2021;9:1402.

15. Hanley JA, McNeil BJ. The meaning and use of the area under a receiver operating characteristic (ROC) curve. Radiology 1982;143:29-36.
16. Wolbers M, Koller MT, Witteman JC, et al. Prognostic models with competing risks: methods and application to coronary risk prediction. Epidemiology 2009;20:555-61.

17. Coutant C, Olivier C, Lambaudie E, et al. Comparison of models to predict nonsentinel lymph node status in breast cancer patients with metastatic sentinel lymph nodes: a prospective multicenter study. J Clin Oncol 2009;27:2800-8.

18. Vickers AJ, Elkin EB. Decision curve analysis: a novel method for evaluating prediction models. Med Decis Making 2006;26:565-74.

19. Giuffrida AY, Burgueno JE, Koniaris LG, et al. Chondrosarcoma in the United States (1973 to 2003): an analysis of 2890 cases from the SEER database. J Bone Joint Surg Am 2009;91:1063-72.

20. Andreou D, Ruppin S, Fehlberg S, et al. Survival and prognostic factors in chondrosarcoma: results in 115 patients with long-term follow-up. Acta Orthop 2011;82:749-55.

21. Jawad MU, Haleem AA, Scully SP. Malignant sarcoma of the pelvic bones: treatment outcomes and prognostic factors vary by histopathology. Cancer 2011;117:1529-41.

22. van Maldegem AM, Gelderblom H, Palmerini E, et al. Outcome of advanced, unresectable conventional central chondrosarcoma. Cancer 2014;120:3159-64.

23. Song K, Shi X, Wang H, et al. Can a Nomogram Help to Predict the Overall and Cancer-specific Survival of Patients With Chondrosarcoma? Clin Orthop Relat Res 2018;476:987-96.

24. Song K, Shi X, Liang X, et al. Risk factors for metastasis at presentation with conventional chondrosarcoma: a population-based study. Int Orthop 2018;42:2941-8.

25. Nguyen MT, Jiang YQ, Li XL, et al. Risk Factors for Incidence and Prognosis in Chondrosarcoma Patients with Pulmonary Metastasis at Initial Diagnosis. Med Sci Monit 2019;25:10136-53.

26. Zhou ZR, Wang WW, Li Y, et al. In-depth mining of clinical data: the construction of clinical prediction model with R. Ann Transl Med 2019;7:796.

27. Song K, Song J, Shi X, et al. Development and Validation of Nomograms Predicting Overall and Cancer-Specific Survival of Spinal Chondrosarcoma Patients. Spine (Phila Pa 1976) 2018;43:E1281-9.

28. Zhang J, Pan Z, Zhao F, et al. Development and validation of a nomogram containing the prognostic determinants of chondrosarcoma based on the Surveillance, Epidemiology, and End Results database. Int J Clin Oncol 2019;24:1459-67. 
29. Thorkildsen J, Taksdal I, Bjerkehagen B, et al. Risk stratification for central conventional chondrosarcoma of bone: A novel system predicting risk of metastasis and death in the Cancer Registry of Norway cohort. J Surg Oncol 2020;121:1115-25.

30. Puri A, Shah M, Agarwal MG, et al. Chondrosarcoma of bone: does the size of the tumor, the presence of a pathologic fracture, or prior intervention have an impact on local control and survival? J Cancer Res Ther 2009; 5:14-9.

31. Shi RL, Qu N, Lu ZW, et al. The impact of marital status at diagnosis on cancer survival in patients with differentiated thyroid cancer. Cancer Med 2016;5:2145-54.

32. Chen Z, Yin K, Zheng D, et al. Marital status

Cite this article as: Hoang TM, Nguyen MT, Chen W, Zhuang C, Wang Z, Wang H, Li J, Lin H. Establishment and validation of an individualized nomogram to predict distant metastasis in chondrosarcoma patients: a population-based study. Transl Cancer Res 2022;11(2):327-338. doi: 10.21037/tcr21-2212 independently predicts non-small cell lung cancer survival: a propensity-adjusted SEER database analysis. J Cancer Res Clin Oncol 2020;146:67-74.

33. Zhou YJ, Lu XF, Zheng KI, et al. Marital status, an independent predictor for survival of gastric neuroendocrine neoplasm patients: a SEER database analysis. BMC Endocr Disord 2020;20:111.

34. Gao Z, Ren F, Song H, et al. Marital Status and Survival of Patients with Chondrosarcoma: A Population-Based Analysis. Med Sci Monit 2018;24:6638-48.

35. Li W, Dong S, Wang H, et al. Risk analysis of pulmonary metastasis of chondrosarcoma by establishing and validating a new clinical prediction model: a clinical study based on SEER database. BMC Musculoskelet Disord 2021;22:529. 